\title{
Sleep disturbances in pediatric bipolar disorder: a comparison between bipolar I and bipolar NOS
}

\author{
Argelinda Baroni ${ }^{1,2}$ *, Mariely Hernandez ${ }^{1,3}$, Marie C. Grant ${ }^{1}$ and Gianni L. Faedda ${ }^{1,2}$ \\ ${ }^{1}$ Mood Disorders Center "Lucio Bini," New York, NY, USA \\ ${ }^{2}$ Department of Child and Adolescent Psychiatry, New York University, New York, NY, USA \\ ${ }^{3}$ Department of Clinical Psychology, Teachers College, Columbia University, New York, NY, USA
}

\section{Edited by:}

Pratibha N. Reebye, British

Columbia's Children's Hospital,

Canada

Reviewed by:

David Matuskey, Yale University

School of Medicine, USA

Bashkim Kadriu, Albert Einstein

College of Medicine of Yeshiva

University, USA

*Correspondence:

Argelinda Baroni, 245 East 50th

Street, Suite 2A, New York, NY

10022-7752, USA.

e-mail: argelinda.baroni @nyumc.org.

\begin{abstract}
Introduction: The diagnosis of bipolar disorder (BD) in youths has been controversial, especially for the subtype BD not otherwise specified (BD-NOS). In spite of growing evidence that sleep is a core feature of $\mathrm{BD}$, few studies characterize and compare sleep disturbances in youth with BD type I (BD-I) and BD-NOS. Sleep disturbances are frequently reported in clinical descriptions of children and adolescents with $\mathrm{BD}$, however the reporting of the frequency and characteristics of sleep symptoms in youth with BD-NOS and BD-I during episodes remain poor. This study compares symptom of sleep disturbance as occurring in manic and depressive episodes in BD-I and BD-NOS youth using Kiddie-schedule for affective disorders and schizophrenia, present and lifetime version (K-SADS-PL) interview data. The study also addresses whether symptoms of sleep disturbance vary in different age groups. Materials and Methods: The sample consisted of 70 children and adolescent outpatients at an urban specialty clinic $(42 \mathrm{M} / 28 \mathrm{~F}, 10.8 \pm 3.6$ years old) including 24 BD-I and 46 BD-NOS assessed using K-SADS-PL-parent interview. Results: Sleep disturbances including insomnia and decreased need for sleep were reported by $84.3 \%$ of the sample. Enuresis was diagnosed in $27 \%$ of sample. There were no significant differences in frequency of sleep symptoms between BD-I and BD-NOS. Regardless of BD subtype, current functioning was negatively correlated with decreased need for sleep but not insomnia, and regardless of BD subtype. Conclusion: The majority of youth with BD presents with sleep symptoms during mood episodes. BD-NOS presents with the same proportion of sleep symptoms as BD-I in our sample.
\end{abstract}

Keywords: adolescent, bipolar disorder, child, sleep, enuresis

\section{INTRODUCTION}

Bipolar disorder (BD), is a chronic illness with a lifetime prevalence (for BD-I, II and not otherwise specified, NOS; DSM-IV) estimated in the US at 4.4\% (Merikangas et al., 2007). A recent meta-analysis indicates a rate of $1.8 \%$ in children and adolescents across 12 studies (Van Meter et al., 2011).

Sleep disturbances are frequent in patients with BD and occur during all phases of $\mathrm{BD}$ as well as during periods of remission (Wehr et al., 1987; Boivin, 2000; Jackson et al., 2003; Harvey et al., 2005; Salvatore et al., 2008). Sleep disturbances might be crucial to the pathogenesis progression and treatment, including relapse prevention. Accordingly, pharmacological interventions routinely target sleep disturbances, while sleep education and hygiene have been successfully integrated with other relapse prevention and maintenance treatment for patients with BD (Frank et al., 2000; Harvey, 2009).

Although there are published reports on sleep in adults with $\mathrm{BD}$, data on the characteristics of sleep disturbances in children with BD and their frequency is still minimal (Harvey, 2009).

For several years the diagnosis of $\mathrm{BD}$ in youths has been controversial because the clinical features might seem inconsistent with the adult presentation of $\mathrm{BD}$. Pediatric $\mathrm{BD}$ often presents with symptoms of fluctuating intensity and duration, often too short to be classified as "episodes" (Faedda et al., 1995, 2004; Geller et al., 1995; Egeland et al., 2000). For those children that do not meet full "adult criteria," including clear episodicity, duration criteria, and number of symptoms, the American Academy of Child and Adolescent Psychiatry recommends the diagnosis of BD-NOS (McClellan et al., 2007). Failure to meet DSM-IV duration criteria is the most common reason for a child not being diagnosed as BDI or II and thus being classified as BD-NOS (Axelson et al., 2006). There is clear evidence that BD-NOS children are as impaired as those with BD-I, despite not meeting full DSM-IV criteria for Mania. Up to $45 \%$ of the BD-NOS cases cross the threshold to BD-I or -II in a median time span of 58 months (Axelson et al., 2006, 2011).

Like adults, children with mania have a "decreased need for sleep" and this feature has been indicated as a cardinal symptom of pediatric BD (Geller et al., 2002). Decreased need for sleep is one of the DSM criteria for mania, with rates ranging from 43 to $72 \%$ (Axelson et al., 2006). Only a few studies to date have examined other sleep disturbances besides decreased need for sleep in children diagnosed with BD (Lewinsohn et al., 1995; Findling et al., 2001; Geller et al., 2002, 2004; Faedda et al., 2004; Faedda and 
Teicher, 2005; Kowatch et al., 2005; Lofthouse et al., 2007, 2008, 2010; Staton et al., 2008a; Harvey, 2009).

In fact, clinically prominent sleep disturbances are common in pediatric $\mathrm{BD}$, occurring in all phases of illness, during remission and subsyndromal periods. Manic, mixed, and depressive phases or rapid-cycling BD cause severe sleep disturbances besides reduced need for sleep, including early, middle, and late insomnia as well as parasomnias (Popper, 1990; Papolos and Papolos, 1997; Faedda et al., 2004; Faedda and Teicher, 2005; Lofthouse et al., 2007; Papolos et al., 2007; Staton, 2008).

Clinically significant sleep disturbances and parasomnias were reported by parents in $95.1 \%$ of 82 outpatient youths ( $4-17$ year old) with BD (Faedda et al., 2004).

Parental reports in 133 children with BD indicated that $96.2 \%$ suffered with moderate to severe sleep problems related to manic, depressive, or comorbid symptoms, but especially during mixed bipolar states (Lofthouse et al., 2007). In a web-based survey of parents of children with BD, Lofthouse et al. $(2008,2010)$ reported that nearly all children (96.9\%) were impaired by sleep difficulties as rated by the Children's Sleep Habits Questionnaire (CSHQ; Owens et al., 2000), requiring pharmacological or non-pharmacological interventions.

Staton (2008) determined that sleep disturbances occurred in $84.5 \%$ of 130 consecutively assessed 3-17 year olds with BD diagnosed by semi-structured interview using modified DSM-IV criteria. Sleep disturbances included evening activation with early insomnia, difficulty with morning awakening with or without decreased need for sleep.

Despite the central role of sleep in $\mathrm{BD}$, there is still sparse data in the pediatric population (Harvey, 2009) and few studies address BD-NOS. For these reasons, this study describes a sample of BD-I and BD-NOS with regard to sleep symptoms during mood episodes and whether sleep dimensions correlate with global functioning.

\section{MATERIALS AND METHODS SUBJECTS}

Children (age 5-17) with BD were recruited from consecutive referrals to the Mood Disorders Center, a private outpatient specialty clinic in New York, NY. Direct interviews by a psychiatrist (GLF) with the parent/guardian and the child were scheduled. The research protocol was approved by Liberty Institutional Review Board, DeLand, FL. The parents of patients gave oral and written informed consent for participation in the evaluation. Children were evaluated by direct observation and interview, as well as interview with their parents, other professionals and teachers involved in the child's care. Available medical records were reviewed. The subjects were recruited between March 2000 and May 2011.

\section{DIAGNOSTIC ASSESSMENT}

Parents were administered a semi-structured interview, the Kiddieschedule for affective disorders and schizophrenia, present and lifetime version (K-SADS-PL, Kaufman et al., 1997). The interview was administered by trained master-level clinicians to assess primary diagnoses. Medicated and unmedicated children were included into the study. The K-SADS assessments begin with a semi-structured interview that reviews medical conditions, lifetime treatment history and course of illness. To establish the diagnoses we used the most severe episodes of depressive and manic symptoms in the subjects' lifetime. As per the K-SADS protocol, mood symptoms that are in common with other psychiatric disorders (such as motor hyperactivity or distractibility) are not rated as present in the mood sections unless they intensify with the onset of abnormal mood. Subjects that met DSM-IV criteria for mania received the diagnosis of BD-I, those with hypomania and Major Depression were diagnosed as BP-II. Of those who did not meet criteria for BD-I or BD-II, all had either, a distinct period of abnormally elevated, expansive, or irritable mood plus 2 or more DSM-IV manic symptoms, a clear change in functioning, or a sufficient number of symptoms that did not meet duration (1 week) or severity criteria for BD-I. All these cases were classified as BD-NOS. Moreover subjects with hypomania without major depressive episodes were also classified as BD-NOS as per DSMIV. We grouped subjects with cyclothymic disorder and BD-NOS, given the difficulty in consistently assessing by retrospective report the absence of 2 months of euthymic mood.

Subjects with a lifetime diagnoses of schizophrenia, autistic spectrum disorders, mental retardation, or mood disorders secondary to a medical condition, substance abuse, or mood disorder due to the use of a medication, were excluded.

Sleep variables were obtained from the K-SADS-PL mood and voiding disorders modules. Sleep symptoms are recorded within mood episodes accordingly to DSM-IV. The variables used in the analyses were: decreased need for sleep, repeated nighttime voiding (nocturnal enuresis), initial insomnia, middle insomnia, terminal insomnia, and circadian reversal.

The subject's primary caretaker was interviewed about his or her personal psychiatric history and about the psychiatric status of the subject's first- and second-degree relatives. Family psychiatric history was rated "positive" if at least one first- or second-degree relative had a diagnosed or clearly diagnosable mood, substanceuse disorder, or a history of suicidal attempt or completed suicide. Socio-economic status was measured using the Hollingshead four-factor scale (Hollingshead, 1975).

\section{ANALYSIS}

A commercially available software program (SPSS Release 16.0 for Windows; SPSS Inc., Chicago, IL, USA) was used for data analysis. The data analyzed for this report were gathered as detailed above. Differences in demographic factors between the two BD subtypes were analyzed using Chi-Square and $t$-Student analyses.

For the factors for which potential significant differences existed among the groups, models were constructed, controlling for the demographic or clinical factors (as medication status) that might have affected the results.

In this exploratory analysis, both threshold and sub-threshold sleep symptoms were recorded as present, and analyzed as a whole, with different types of insomnia (initial, middle, and terminal) grouped together to obtain gross estimates of any sleep disturbance. To investigate similarities in sleep disturbance between $\mathrm{BD}-\mathrm{I}$ and BD-NOS, as well as test the importance of sleep disturbance to current functioning for both disorders, analyses of variance were run to assess whether sleep disturbance symptoms affected current functioning in both BD-I and BD-NOS subjects. 


\section{RESULTS}

The initial cohort included 99 subjects with a K-SADS-PL diagnosis. We excluded from the analysis four cases with BD-II, nine cases with Major Depressive Disorder, nine cases with other primary lifetime diagnoses, and four cases that were younger than 5 years old at the time of the interview.

We report here on the 70 children with a K-SADS-PL diagnosis of BD-I or BD-NOS. The sample consisted of 42 males and 28 females, with mean age at interview $10.8 \pm 3.6$ years old. The sample included $24 \mathrm{BD}-\mathrm{I}$, and $46 \mathrm{BD}-\mathrm{NOS}$. Most of the sample was Caucasian (88.6), had a high socio-economic status $(85.7 \%$ between 40 and 66 for Hollingshead scale), 14.3\% were adopted and $65.7 \%$ were assuming psychotropic medications at the time of the K-SADS interview. More than $92 \%$ had a positive family history for a psychiatric disorder.

Sleep symptoms were extremely common, with $84.3 \%$ of subjects experiencing at least one sleep symptom during (within) a mood episode. This number includes $71.4 \%$ with insomnia during depressive episodes, and 51.4\% experiencing decreased need for sleep during mania/hypomania. Additionally, 22\% reported circadian reversal and $27 \%$ had nocturnal enuresis. There were no statistically significant differences in gender distribution, age, ethnicity, and socio-economic statuses or in any of the sleep symptoms except for enuresis, with a higher prevalence in BD-NOS. After controlling for medication status, the difference in enuresis lost significance as well.

Demographic information for the subgroups sample can be found in Table 1.

Frequencies of sleep disturbances for the two subgroups including enuresis, insomnia, and decreased need for sleep are illustrated in Table 2.

Among subjects with BD-I $(n=24)$, results showed significant differences in current functioning between subjects with versus without a decreased need for sleep $(p<0.05)$. Specifically, BD-I subjects with a decreased need for sleep showed significantly

Table 1 | Demographic information.

\begin{tabular}{|c|c|c|c|c|}
\hline & BD-I & BD-NOS & Total & Sig. \\
\hline$N(\%)$ & $24(34.3)$ & $46(65.7)$ & $70(100)$ & \\
\hline Age mean yrs (SD), range 5-17 & $10.5 \pm 3.7$ & $11 \pm 3.5$ & $10.8 \pm 3.6$ & N.S. \\
\hline Gender, male & $15(62.5)$ & $27(58.7)$ & $42(60)$ & N.S. \\
\hline \multicolumn{5}{|l|}{ SES } \\
\hline $20-29$ & $0(0)$ & $0(0)$ & $0(0)$ & N.S. \\
\hline 30-39 & $3(12.5)$ & $7(15.2)$ & $10(14.3)$ & \\
\hline $40-54$ & $7(29.2)$ & $18(39.1)$ & $25(35.7)$ & \\
\hline $55-66$ & $14(58.3)$ & $21(45.7)$ & $35(50)$ & \\
\hline \multicolumn{5}{|l|}{ ETHNICITY } \\
\hline Caucasian & $20(83.3)$ & $42(91.3)$ & $62(88.6)$ & N.S. \\
\hline African Am & $1(4.2)$ & $1(2.2)$ & $2(2.9)$ & \\
\hline Asian & $1(4.2)$ & $0(0)$ & $1(1.4)$ & \\
\hline Biracial & $2(8.3)$ & $2(4.3)$ & $4(5.7)$ & \\
\hline Other & $0(0)$ & $1(2.2)$ & $1(1.4)$ & \\
\hline Adopted, yes & $3(12.5)$ & $7(15.2)$ & $10(14.3)$ & N.S. \\
\hline Positive family history, yes & $22(91.7)$ & $43(93.5)$ & 65 (92.9) & N.S. \\
\hline Medicated, yes & $17(70.8)$ & $29(63)$ & $46(65.7)$ & N.S. \\
\hline
\end{tabular}

worse functioning than those without a decreased need for sleep. Among subjects with BD-NOS $(n=46)$, results showed a similar trend, but did not reach statistical significance $(p=0.096)$. Thus, BD-NOS subjects showed a similar pattern to that in BDI subjects, as subjects with a decreased need for sleep showed significantly worse functioning than those without a decreased need for sleep. Taken together, findings suggest that both subjects with BD-NOS and BD-I are characterized by sleep disturbance (i.e., decreased need for sleep) and impairment in sleep parameters mediates impairment in functioning regardless of diagnostic subtype, further supporting the similarities between BD-NOS and BD-I.

\section{DISCUSSION}

The relationship between sleep disturbances and mood syndromes remains unclear and data on sleep variables early in the course of BD is greatly needed. Sleep disturbances and disorders are an important component of Mood Disorders regardless of polarity and subtype. Moreover the diagnostic debate about children and adolescent with BD-NOS demands more data on this group. In our sample we found:

(1) No differences in sleep symptoms during mood episodes between $\mathrm{BD}-\mathrm{NOS}$ and $\mathrm{BD}-\mathrm{I}$, with both groups showing high frequencies of sleep disturbances during both manic and depressive episodes. The two groups did not differ for type of sleep symptoms during episodes, showing comparable proportions of insomnia and circadian reversal during depressive episodes.

(2) Both groups presented comparable frequencies of decreased need for sleep during manic episodes.

(3) Age did not affect the rate and type of sleep disturbances, and symptoms were equally present in children and adolescents, except for higher presence of circadian reversal in adolescents, as developmentally expected (Crowley et al., 2007).

(4) High rates of enuresis have been described in BD-I and enuresis was more prevalent in our BD-I sample, however when enuresis was controlled for medication status, the statistical significance disappeared, suggesting a possible effect of medication on nocturnal voiding (Klages et al., 2005; Henin et al., 2007). The relationship between medication and enuresis has

\section{Table 2 | K-SADS symptoms by diagnosis.}

\begin{tabular}{lllll}
\hline K-SADS-PL symptoms & $\begin{array}{l}\text { BD-I } \\
\mathbf{N}(\%)\end{array}$ & $\begin{array}{l}\text { BD-NOS } \\
\mathbf{N ~ ( \% )}\end{array}$ & $\begin{array}{l}\text { Total } \\
\mathbf{N ~ ( \% )}\end{array}$ & Sig. \\
\hline Any sleep disorder & $20(83.3)$ & $39(84.8)$ & $59(84.3)$ & N.S. \\
Decreased need for sleep & $13(54.2$ & $23(50)$ & $36(51.4)$ & N.S. \\
Any insomnia & $16(66.7)$ & $34(73.9)$ & $50(71.4)$ & N.S. \\
Circadian reversal & $4(16.7)$ & $12(26.1)$ & $16(22.9)$ & N.S. \\
Nocturnal enuresis & $10(41.7)$ & $9(19.6)$ & $19(27.1)$ & N.S.* \\
Current CGAS & 41.8889 & 41.7083 & & N.S. \\
Most severe CGAS & 36.6304 & 36.0417 & & N.S. \\
Highest CGAS & 41.9778 & 42.0833 & & N.S.
\end{tabular}

*Non-significant when accounted for medication status. 
been previously reported for adult and pediatric psychiatric patients (Kandil et al., 2004; Harrison-Woolrych et al., 2011), but it would deserve more attention in pediatric BD to clarify the causality of this phenomenon.

(5) Measures of global functioning were significantly correlated with "decreased need for sleep" but not with "insomnia," possibly indicating that some manic symptoms are more impairing in younger population then depressive ones.

(6) The BD-I and BD-NOS subjects showed a positive correlation between decreased need for sleep and impairment, as subjects with decreased need for sleep had significantly worse functioning than those without.

Such pervasive sleep disturbances suggest that pediatric BDI - and to same extent; BD-NOS - is similar to adult BD and to other primary mood disorders. Both subjects with BD-NOS and BD-I are characterized by sleep disturbance, but only one (decreased need for sleep) results in impaired functioning, and this is found both in BD-I and in BD-NOS. However, it is unclear with the present data what mediates this association between manic sleep symptoms and functioning - e.g., inattention or poor judgment - within episodes.

Also, our data suggest that there are no differences in proportion of sleep symptoms displayed by youth with BD-NOS and BD-I, and that younger patients present sleep disturbances in the same proportion as older patients with the exclusion of circadian reversal. Of course, by definition, BD-NOS do not meet criteria for symptoms duration or number of symptoms but do present qualitatively the same symptoms.

We also confirmed the higher prevalence of enuresis in patients with $\mathrm{BD}$, and suspect it might be increased both because of high comorbidity and pharmacological treatment of BD.

Lastly, sleep disturbances appear to be a frequent and important feature of $\mathrm{BD}$ early in the course of the illness. Recognizing their prevalence may lead to earlier and more aggressive treatment, possibly improving future course of the illness, socio-emotional development, and academic functioning, in the

\section{REFERENCES}

Axelson, D., Birmaher, B., Strober, M., Gill, M. K., Valeri, S., Chiappetta, L., and Keller, M. (2006). Phenomenology of children and adolescents with bipolar spectrum disorders. Arch. Gen. Psychiatry 63, 1139-1148.

Axelson, D., Birmaher, B., Strober, M., Goldstein, B., Ha, W., Gill, M. K., and Keller, M. (2011). Course of subthreshold bipolar disorder in youth: diagnostic progression from bipolar disorder not otherwise specified. $J$. Am. Acad. Child Adolesc. Psychiatry 50, 1001-1016.

Boivin, D. (2000). Influence of sleepwake and circadian rhythm disturbances in psychiatric disorders. $J$. Psychiatry Neurosci. 25, 446-458.

Crowley, S., Acebo, C., and Carskadon, M. (2007). Sleep, circadian rhythms, and delayed phase in adolescence. Sleep Med. Res. 8, 602-612.
Egeland, J., Hostetter, A., Pauls, D., and Sussex, J. (2000). Prodromal symptoms before onset of manicdepressive disorder suggested by first hospital admission histories. J. Am. Acad. Child Adolesc. Psychiatry 39. 1245-1252.

Faedda, G., Baldessarini, R., Glovinsky, I., and Austin, N. (2004). Pediatric bipolar disorder: phenomenology and course of illness. Bipolar Disord. 6, 305-313.

Faedda, G., Baldessarini, R., Suppes, T., Tondo, L., Becker, I., and Lipschitz, D. (1995). Pediatriconset bipolar disorder: a neglected clinical and public health problem. Harv. Rev. Psychiatry 3, 171-195.

Faedda, G., and Teicher, M. (2005). Objective measures of activity and attention in the differential diagnosis of psychiatric disorders of

same way sleep hygiene positively affects the course of $\mathrm{BD}$ in adults.

\section{LIMITATIONS}

Several limitations relating to the preliminary nature of the data presented must be underscored, as they require replication in controlled, prospective studies.

Cases were recruited from a private outpatient clinic, and the K-SADS was offered in an open enrollment, non-randomized, voluntary basis; therefore the sample is not necessarily representative of all BD cases. Moreover, while the K-SADS is considered the gold standard for diagnostic assessment, the diagnosis and the symptoms collection are often based on retrospective data. While retrospective interviews are always subject to bias, given the young age of our population, we expect that the time elapsed from the symptoms and the interview is brief or relatively brief, thus minimizing possible errors. Since we did not collect the age at syndromal onset we cannot quantify the interval between data collection and onset of BD. Also, children and adolescent cases were assigned based on their age rather than a screening or physical assessment of their pubertal status.

Kiddie-schedule for affective disorders and schizophrenia, present and lifetime version interviews were administered to parents or caretakers, but not to the children and adolescents. This could potentially lead to an underestimate of sleep symptoms rather than an overestimation, as parents might not be aware of insomnia or decreased need for sleep in their child. However lifetime diagnoses were obtained by structured interview according to DSM-IV criteria, and confirmed by clinical diagnosis and course of illness.

We focused solely on BD-I and NOS, not addressing the full spectrum of the illness and did not differentiate between cyclothymic disorder and BD-NOS.

In this preliminary study, we did not administer rating scales to assess phase of illness and severity of symptoms, so we are not able to assess correlation between current symptoms' severity and abnormalities of sleep.

childhood. Essent. Psychopharmacol. 6, 239-249.

Findling, R., Gracious, B., McNamara, N., Youngstrom, E., Demeter, C., and Calabrese, J. (2001). Rapid, continuous cycling and psychiatric comorbidity in pediatric bipolar I disorder. Bipolar Disord. 3, 202-210.

Frank, E., Swartz, H., and Kupfer, D. (2000). Interpersonal and social rhythm therapy: managing the chaos of bipolar disorder. Biol. Psychiatry 48, 593-604.

Geller, B., Sun, K., Zimerman, B., Luby, J., Frazier, J., and Williams, M. (1995). Complex and rapid-cycling in bipolar children and adolescents: a preliminary study. J. Affect. Disord. 34, 259-268.

Geller, B., Tillman, R., Craney, J., and Bolhofner, K. (2004). Four-year prospective outcome and natural history of mania in children with a prepubertal and early adolescent bipolar disorder phenotype. Arch. Gen. Psychiatry 61, 459-467.

Geller, B., Zimerman, B., Williams, M., DelBello, M., Frazier, J., and Beringer, L. (2002). Phenomenology of prepubertal and early adolescent bipolar disorder: examples of elated mood, grandiose behaviors, decreased need for sleep, racing thoughts and hypersexuality. J. Child Adolesc. Psychopharmacol. 12, 3-9.

Harrison-Woolrych, M., Skegg, K., Ashton, J., Herbison, P., and Skegg, D. (2011). Nocturnal enuresis in patients taking clozapine, risperidone, olanzapine and quetiapine: comparative cohort study. Br. J. Psychiatry 199, 140-144.

Harvey, A. (2009). The adverse consequences of sleep disturbance in pediatric bipolar disorder: implications 
for intervention. Child Adolesc. Psychiatr. Clin. N. Am. 48, 593-604.

Harvey, A., Schmidt, D., Scarna, A., Semler, C., and Goodwin, G. (2005). Sleep-related functioning in euthymic patients with bipolar disorder, patients with insomnia, and subjects without sleep problems. Am. J. Psychiatry 162, 50-57.

Henin, A., Biederman, J., Mick, E., Hirshfeld-Becker, D., Sachs, G., Wu, Y., Yan, L., Ogutha, J., and Nierenberg, A. (2007). Childhood antecedent disorders to bipolar disorder in adults: a controlled study. J. Affect. Disord. 99, 51-57.

Hollingshead, A. B. (1975). Four-Factor Index of Social Status. New Haven, Conn: Sociology Dept, Yale University.

Jackson, A., Cavanagh, J., and Scott, J. (2003). A systematic review of manic and depressive prodromes. J. Affect. Disord. 74, 209-217.

Kandil, S., Aksu, H., and Ozyavuz, R. (2004). Reversible nocturnal enuresis in children receiving SSRI with or without risperidone: presentation of five cases. Isr. J. Psychiatry Relat. Sci. 41, 218-221.

Kaufman, J., Birmaher, B., Brent, D., Rao, U., Flynn, C., and Moreci, P. (1997). Schedule for affective disorders and schizophrenia for schoolage children - present and lifetime version (KSADS-PL): initial reliability and validity data. J. Am. Acad. Child Adolesc. Psychiatry 36, 980-988.

Klages, T., Geller, B., Tillman, R., Bolhofner, K., and Zimerman, B. (2005). Controlled study of encopresis and enuresis in children with a prepubertal and early adolescent bipolar-I disorder phenotype. J. Am. Acad. Child Adolesc. Psychiatry 44, 1050-1057.

Kowatch, R., Youngstrom, E., Danielyan, A., and Findling, R. (2005). Review and meta-analysis of the phenomenology and clinical characteristics of mania in children and adolescents. Bipolar Disord. 7, 483-496.

Lewinsohn, P., Klein, D., and Seeley, J. (1995). Bipolar disorders in a community sample of older adolescents: prevalence, phenomenology, comorbidity, and course. J. Am. Acad. Child Adolesc. Psychiatry 34, 454-463.

Lofthouse, N., Fristad, M., Splaingard, M., and Kelleher, K. (2007). Parent and child reports of sleep problems associated with early-onset bipolar spectrum disorders. J. Fam. Psychol. 21, 114-123.

Lofthouse, N., Fristad, M., Splaingard, M., Kelleher, K., Hayes, J., and Resko, S. (2008). Web survey of sleep problems associated with early-onset bipolar spectrum disorders. J. Pediatr. Psychol. 33, 349-357.

Lofthouse, N., Fristad, M., Splaingard, M., Kelleher, K., Hayes, J., and Resko, S. (2010). Web-survey of pharmacological and non-pharmacological sleep interventions for children with early-onset bipolar spectrum disorders. J. Affect. Disord. 120, 267-271.

McClellan, J., Kowatch, R., and Findling, R. (2007). Practice parameter for the assessment and treatment of children and adolescents with bipolar disorder. J. Am. Acad. Child Adolesc. Psychiatry 46, 107-125.

Merikangas, K., Akiskal, H., Angst, J., Greenberg, P., Hirschfeld, R.,
Petukhova, M., and Kessler, R. (2007). Lifetime and 12-month prevalence of bipolar spectrum disorder in the National Comorbidity Survey replication. Arch Gen. Psychiatry 64, 543-552. [Erratum in: Archives of General Psychiatry. 2007 Sep; 64(9): 1039.1].

Owens, J., Spirito, A., and McGuinn, M. (2000). The Children's Sleep Habits Questionnaire (CSHQ): psychometric properties of a survey instrument for school-aged children. Sleep 23 1043-1051.

Papolos, D., Hennen, J., Cockerham, M., and Lachman, H. (2007). A strategy for identifying phenotypic subtypes: concordance of symptom dimensions between sibling pairs who met screening criteria for a genetic linkage study of childhood-onset bipolar disorder using the Child Bipolar Questionnaire. J. Affect. Disord. 99, 27-36.

Papolos, D., and Papolos, J. (1997). The Bipolar Child. New York: Broadway Books.

Popper, C. (1990). On diagnostic gore in child's nightmares. American Academy of Child and Adolescent Psychiatry Newsletter, 3-4.

Salvatore, P., Ghidini, S., Zita, G., De Panfilis, C., Lambertino, S., Maggini, C., and Baldessarini, R. (2008). Circadian activity rhythm abnormalities in ill and recovered bipolar I disorder patients. Bipolar Disord. 10, 256-265.

Staton, D. (2008). The impairment of pediatric bipolar sleep: hypotheses regarding a core defect and phenotype-specific sleep disturbances. J. Affect. Disord. 108, 199-206.

Staton, D., Volness, L., and Beatty, W. (2008a). Diagnosis and classification of pediatric bipolar disorder. $J$. Affect. Disord. 105, 205-212.

Van Meter, A. R., Moreira, A. L., and Youngstrom, E. A. (2011). Metaanalysis of epidemiological studies of pediatric bipolar disorder. J. Clin. Psychiatry 72, 1250-1256.

Wehr, T., Sack, D., and Rosenthal, N. (1987). Sleep reduction as a final common pathway in the genesis of mania. Am. J. Psychiatry 144, 201204 (with corrections, 144, 542).

Conflict of Interest Statement: Gianni L. Faedda, M.D. Receives royalties from the book "Parenting a Bipolar Child," New Harbinger Publications, Oakland CA, USA, 2006.

Received: 13 November 2011; accepted: 01 March 2012; published online: 20 March 2012.

Citation: Baroni A, Hernandez M, Grant MC and Faedda GL (2012) Sleep disturbances in pediatric bipolar disorder: a comparison between bipolar I and bipolar NOS. Front. Psychiatry 3:22. doi: 10.3389/fpsyt.2012.00022

This article was submitted to Frontiers in Child and Neurodevelopmental Psychiatry, a specialty of Frontiers in Psychiatry. Copyright (C) 2012 Baroni, Hernandez, Grant and Faedda. This is an open-access article distributed under the terms of the Creative Commons Attribution Non Commercial License, which permits noncommercial use, distribution, and reproduction in other forums, provided the original authors and source are credited. 